\title{
A Signature of Circulating microRNAs Predicts the Susceptibility of Acute Mountain Sickness
}

\section{OPEN ACCESS}

Edited by:

Gaetano Santulli,

Columbia University, USA

Reviewed by:

Rosalba Giugno,

University of Verona, Italy

Francesco Russo,

University of Copenhagen, Denmark

${ }^{*}$ Correspondence:

Jian Chen

jchenone@163.com

Yu-Qi Gao

gaoy66@yahoo.com

${ }^{\dagger}$ These authors have contributed equally to this work.

Specialty section:

This article was submitted to

Clinical and Translational Physiology,

a section of the journal

Frontiers in Physiology

Received: 21 December 2016

Accepted: 23 January 2017

Published: 08 February 2017

Citation:

Liu B, Huang $H, W u G, X \cup G$, Sun $B-D$, Zhang E-L, Chen J and

Gao Y-Q (2017) A Signature of

Circulating microRNAs Predicts the

Susceptibility of Acute Mountain

Sickness. Front. Physiol. 8:55.

doi: 10.3389/fphys.2017.00055

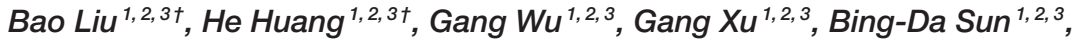 \\ Er-Long Zhang 1, 2,3, Jian Chen 1, 2,3* and Yu-Qi Gao ${ }^{1,2,3 *}$
}

${ }^{1}$ Institute of Medicine and Hygienic Equipment for High Altitude Region, College of High Altitude Military Medicine, Third Military Medical University, Chongqing, China, ${ }^{2}$ Key Laboratory of High Altitude Environmental Medicine, Third Military Medical University, Ministry of Education, Chongqing, China, ${ }^{3}$ Key Laboratory of High Altitude Medicine, PLA, Chongqing, China

Background: Acute mountain sickness (AMS) is a common disabling condition in individuals experiencing high altitudes, which may progress to life-threatening high altitude cerebral edema. Today, no established biomarkers are available for prediction the susceptibility of AMS. MicroRNAs emerge as promising sensitive and specific biomarkers for a variety of diseases. Thus, we sought to identify circulating microRNAs suitable for prediction the susceptible of AMS before exposure to high altitude.

Methods: We enrolled 109 healthy man adults and collected blood samples before their exposure to high altitude. Then we took them to an elevation of $3648 \mathrm{~m}$ for 5 days. Circulating microRNAs expression was measured by microarray and quantitative reverse-transcription polymerase chain reaction (qRT-PCR). AMS was defined as Lake Louise score $\geq 3$ and headache using Lake Louise Acute Mountain Sickness Scoring System.

Results: A total of 31 microRNAs were differentially expressed between AMS and Non-AMS groups, 15 up-regulated and 16 down-regulated. Up-regulation of miR-369-3p, miR-449b-3p, miR-136-3p, and miR-4791 in patients with AMS compared with Non-AMS individuals were quantitatively confirmed using qRT-PCR (all, $P<0.001$ ). With multiple logistic regression analysis, a unique signature encompassing miR-369-3p, miR-449b-3p, and miR-136-3p discriminate AMS from Non-AMS (area under the curve 0.986, 95\% Cl 0.970-1.000, $P<0.001$, LR+: 14.21, LR-: 0.08). This signature yielded a $92.68 \%$ sensitivity and a $93.48 \%$ specificity for AMS vs. Non-AMS.

Conclusion: The study here, for the first time, describes a signature of three circulating microRNAs as a robust biomarker to predict the susceptibility of AMS before exposure to high altitude.

Keywords: circulating microRNAs, signature, prediction, acute mountain sickness

\section{INTRODUCTION}

There are three main highland regions of the world that support large populations, including the Tibetan plateau and Himalayan valleys, the Andes of South America, and the Ethiopian highlands. With the development of economy and tourism, the number of individuals who ascended to high altitude for a variety of reasons is increasing. Acute mountain sickness (AMS) is a public health 
problem of persons who acutely ascended to high altitude with an elevation more than $2500 \mathrm{~m}$ (Hackett et al., 1976; MacInnis et al., 2013; Waeber et al., 2015). It may occur as early as 6$24 \mathrm{~h}$ following ascent (Wright et al., 2008). The primary clinical manifestation of AMS is a combination of several symptoms, such as headache, anorexia, dizziness, malaise, nausea, and sleep disturbance (Bärtsch and Swenson, 2013). Among these symptoms, headache is the cardinal symptom of AMS and is essential to diagnose AMS according to Lake Louise Scoring System (LLS; Roach et al., 1993). LLS is a simple and widely accepted tool for AMS assessment (Maggiorini et al., 1998), which was created at the Hypoxia Symposium in 1991 and later modified at the following Hypoxia Symposium in 1993. The LLS encompasses two parts, a self-report questionnaire including five symptoms (headache, gastrointestinal symptoms, dizziness, and difficulty sleeping) and a clinical assessment comprising three physical examination findings (altered mental status, ataxia, and peripheral edema). Severe AMS could make persons to be incapacitating during the period of exposure to high altitude and, more seriously, developed to high altitude cerebral edema which is a life-threatening form of acute altitude illness (Basnyat and Murdoch, 2003).

Given the high incidence of AMS among travelers and its deleterious effect on health, identifying those susceptible to the disorder would be useful as it would assist in the development of preventive strategies for a given individual. By far several prediction tools have been proposed, including cold pressor test (Kovtun and Voevoda, 2013), heart rate variability (Koehle et al., 2010; Karinen et al., 2012), and lung functions (Zhou et al., 2004). All these tools detected the physiological parameters of body, which were convenient and have low cost. Recently, there was several studies focus on gene features in the screening susceptibility of AMS (Ding et al., 2011). Although, the parameters measured by those above tools present significant association with the risk of AMS. The low sensitivity and specificity limited the use of those simple sea level tests (Song et al., 2013) and gene polymorphism detection method (Ding et al., 2011).

MicroRNAs are small ( $\sim 22-$ nt long), noncoding, single-stranded RNAs that regulate gene expression posttranscriptionally in various physiological and pathophysiological cellular processes such as proliferation (Lenkala et al., 2014), differentiation (Shivdasani, 2006), metabolism (Dumortier et al., 2013), and apoptosis (Su et al., 2015). In recent years, researchers have found that microRNAs are also present in different body fluids, the one in blood refer to circulating microRNA (Weber et al., 2010). Circulating microRNAs are remarkably stable and can be specific to certain physiological and pathological conditions and are accessible to analysis through relatively non-invasive methods (Mitchell et al., 2008). The significant differences in their expression have been described in a wide array of various diseases, although their physiological functions

\footnotetext{
Abbreviations: AMS, acute mountain sickness; qRT-PCR, quantitative reversetranscription polymerase chain reaction; LLS, Lake Louise Scoring System; AUC, area under the receiver-operating characteristic curve; LR, likelihood ratio; NO, nitric oxide; GO, gene ontology.
}

remained unknown (Ai et al., 2010; Moussay et al., 2011; Roth et al., 2011).

Therefore, in the present study, we performed miRCURYTM LNA Array (v.18.0) (Exiqon) screening, followed by data validation with quantitative reverse-transcription polymerase chain reaction (qRT-PCR) to evaluate plasma microRNA profiles of individuals before exposure to high altitude to predict the susceptibility of AMS. Interestingly, our data indicated that a signature combined with circulating microRNAs miR-369-3p (MIMAT0000721), miR-449b-3p (MIMAT0009203), and miR136-3p (MIMAT0004606) provided substantial AMS prediction.

\section{METHODS AND MATERIALS}

\section{Participants}

This study protocol was approved by the Ethics Committee of third Military Medical University, China, in accordance with the Declaration of Helsinki, and all individuals provided written informed consent before entry. Excluding individuals with a history of smoking and high altitude travel, 109 adult health volunteers aged 17-35 years whose primary residence was at an elevation of $1000 \mathrm{~m}$ or lower. Among them, 22 individuals were randomly selected for microRNA array screening, while the remaining 87 constituted the validation set with qRT-PCR method.

\section{Ascent and Blood Samples Collection}

All participants were driven from Chongqing (elevation, $200 \mathrm{~m}$ ), China, before ascent. After a 3 day rest, they acutely ascended to Lhasa (elevation, $3648 \mathrm{~m}$ ), China, by train within $48 \mathrm{~h}$, where they remained for 5 days. Blood samples were collected in EDTA tubes using standard operating procedures in the morning before departure.

During the investigation, all volunteers had the same diet and were required to abstain from strenuous activity to ensure a similar level of physical activity. The investigators continuously monitored the individuals for evidence of high altitude pulmonary edema or high altitude cerebral edema according to the specific clinical manifestations of these diseases (Hackett and Roach, 2004; Pennardt, 2013). Immediate evacuation and treatment with oxygen were available for such an occurrence.

TABLE 1 | Primers used for qRT-PCR verification of differently expressed circulating microRNAs.

\begin{tabular}{lll}
\hline microRnA & Forward primer & Reverse primer \\
\hline miR-369-3p & CAATGGAAATCGAATAATA & TATGCTTGTTCTCGTCTCTGTGTC \\
& CATGG & \\
miR-449b-3p & CGCGCCAGCCACAACTAC & TATGGTTGTCACGACTCCTTCAC \\
miR-136-3p & CGGCGCATCATCGTCTCA & TATGGTTGTCACGACTCCTTCAC \\
miR-4791 & CGCGGCGCTGGATATGA & TATGGTTGTCACGACTCCTTCAC \\
cel-miR-39 & ATATCATCTCACCGGGTGT & TATGGTITGACGACTGTGTGAT \\
& AAATC &
\end{tabular}




\section{Measurements}

We measured main parameters, including blood pressure, heart rate, and blood oxygen saturation, prior to departure and successive 5 days after exposure to high altitude. AMS was diagnosed using the LLS. Self-report questionnaire of LLS were recorded prior to departure and at each day during high altitude exposure (5 days). Meanwhile, the clinical assessment was done by our accompanied doctors. As per the Lake Louise Consensus Group, individuals with a score of three points or greater on the AMS self-report questionnaire alone (including a headache score $\geq 1$ ), or in combination with the clinical assessment score designated as AMS.

\section{RNA Isolation and microRNA Array Analysis}

According to manufacturers' instructions, total RNA was isolated using TRIzol (Invitrogen) and purified with RNeasy mini kit (QIAGEN). RNA quality and quantity were measured on a NanoDrop spectrophotometer (ND-1000, Nanodrop Technologies) and listed in Supplementary Table 1. After quality control, the miRCURY ${ }^{\mathrm{TM}} \mathrm{Hy} 3^{\mathrm{TM}} / \mathrm{Hy}^{\mathrm{TM}}$ Power labeling kit (Exiqon, Vedbaek, Denmark) was used according to the manufacturer's guideline for microRNA labeling. After hybridized $\mathrm{Hy}^{\mathrm{TM}}$-labeled samples on a miRCURYTM LNA Array (v.18.0) (Exiqon) and washed slides several times with a Wash buffer kit (Exiqon), the slides were scanned on an Axon GenePix 4000B microarray scanner (Axon Instruments,

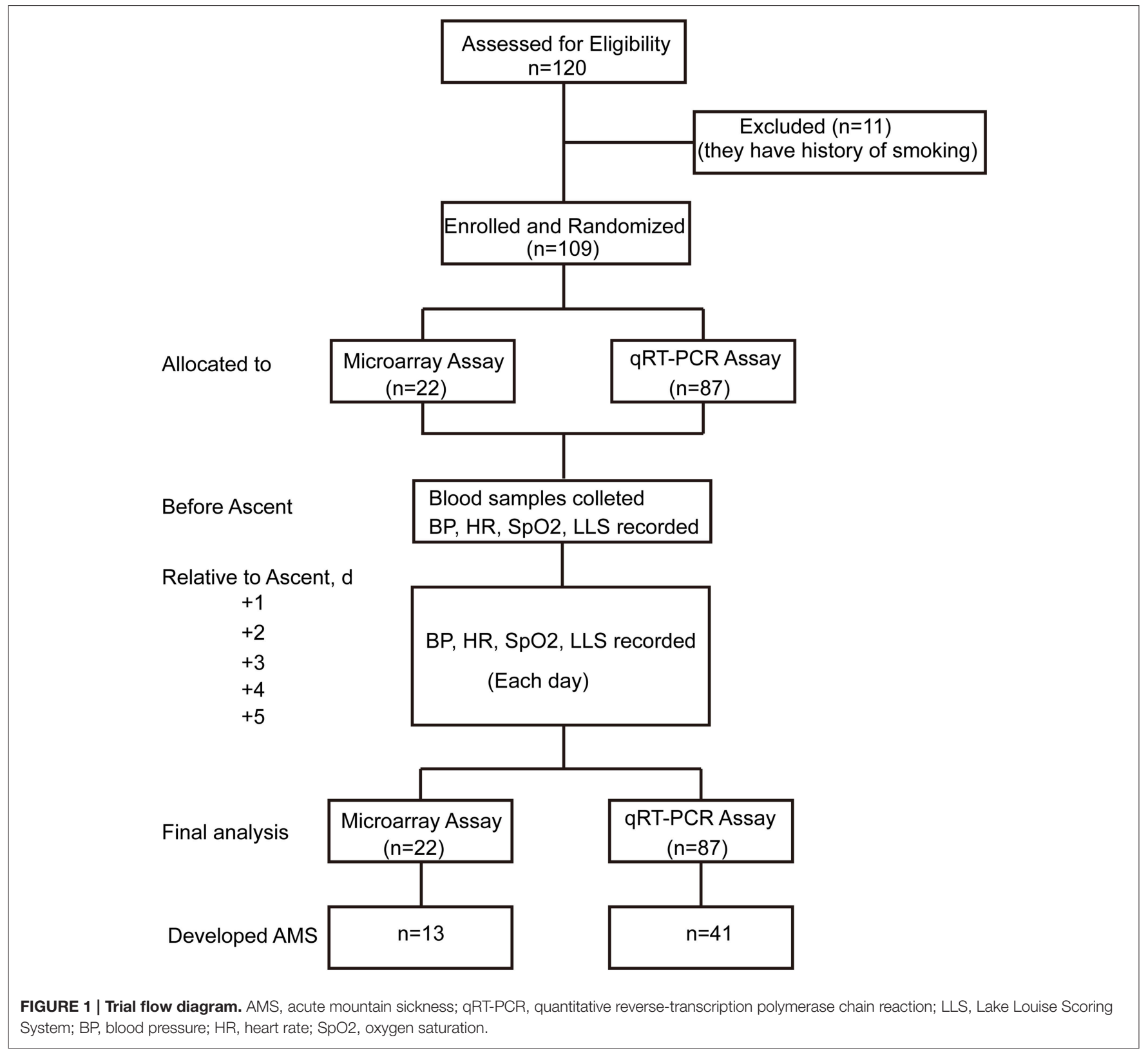


Foster City, CA). Scanned images were then imported into the GenePix Pro 6.0 software (Axon) for grid alignment and data extraction. Replicated microRNAs were averaged, and probes with intensities $\geq 30$ in all samples were selected for normalization. Detailed instructions were as previously described (Liu et al., 2016).After normalization, significantly differentially expressed microRNAs between the two groups were identified using Fold change and $P$-value cutoffs of 2 and 0.05 , respectively.

\section{Quantitative Reverse-Transcription Polymerase Chain Reaction (qRT-PCR)}

For qRT-PCR, a synthetic Caenorhabditis elegans microRNA, cel-miR-39 (Qiagen, Valencia, CA, USA), was added to plasma samples as a control prior to RNA extraction. Total RNA was extracted from $200 \mu \mathrm{L}$ individual plasma samples with a miRNeasy extraction kit (Qiagen, Valencia, CA, USA) according to the manufacturer's instructions. RNA (6 $\mu \mathrm{l})$ was reverse transcribed into cDNA (in a final volume of $10 \mu \mathrm{l}$ ) using a reverse transcription kit (GenePharma, Shanghai, China). Subsequently, quantitative real-time PCR was performed on an $\mathrm{iQ}^{\mathrm{TM}} 5$ RealTime PCR Detection System (Bio-Rid, USA) using SYBR Green. The primers used for qRT-PCR are listed in Table 1. Relative microRNA levels were determined by the $2^{-\Delta C T}$ method.

\section{Identifying Potential Biological Relevance of microRNA Signature}

To explore the potential biological relevance of microRNAs signature, we predicted target genes of microRNAs using microTCDS v5.0 (Paraskevopoulou et al., 2013) and TarBase v7.0 (Vlachos et al., 2015a). microT-CDS is an algorithm which is specifically trained on a positive and a negative set of microRNA recognition elements located in both the $3^{\prime}$-UTR and CDS regions, while TarBase is the largest available manually curated target database, indexing 9-250-fold more entries than any other available database. Both of them are associated with the latest miRBase version (v21) (Akhtar et al., 2016). Gene ontology (GO) enrichment analysis was performed for target genes using DIANA-miRPath v3. 0 which deciphers microRNA function with experimental support (Vlachos et al., 2015b). Network between microRNAs and target genes was constructed by Cytoscape v3.4.0 (Cline et al., 2007).

\section{Statistical Analysis}

Normality was assessed for all datasets by the Shapiro-Wilk's test. Then, independent $t$-test or Mann-Whitney $U$-test was carried out to assess baseline differences between AMS and non-AMS individuals. Comparison of the vitals mean difference between AMS and non-AMS groups using the two-way mixed ANOVA. Receiver operating characteristic curves were analyzed to assess specificity and sensitivity of single-plasma microRNAs and their combination using multiple logistic regression analysis. The optimal diagnostic point of the microRNAs was assessed at cutoff values with the largest Youden's index. To investigate the clinical impact of our signature microRNAs, we have chosen the likelihood ratio (LR) in addition to classical test parameterssensitivity and specificity, because it combines information of both. Odds ratio per standard deviation was calculated by logistic regression analysis. Areas under the receiveroperating characteristic curves (AUCs) were evaluated using Swets classification (Gasparini et al., 2015): AUC $=0.5$, no diagnostic value; $0.5<\mathrm{AUC}<0.7$, accuracy to only a small degree; $0.7<$ AUC $<0.9$, fair accuracy; $0.9<$ AUC $\leq 1$, high accuracy.

Statistical analyses were performed with the $\mathrm{R}$ software (version 3.2.3, R Foundation for Statistical Computing, Vienna, Austria) with package "OptimalCutpoints" (López-Ratón et al., 2014), IBM SPSS Statistics 19 (SPSS, Chicago, IL, USA, and GraphPad Prism 5. The following values were considered significant: $P<0.05\left(^{*}\right)$ and $P<0.01\left(^{* *}\right)$.

\section{RESULTS}

\section{Clinical Manifestation of All Individuals}

The trail flow diagram is shown in Figure 1. Assessed by LLS, 13 individuals were diagnosed as AMS and 9 as Non-AMS in microarray assay, while 41 volunteers were designated as AMS and 46 as Non-AMS in qRT-PCR test. In total, the morbidity of AMS is $49.5 \%$. Respectively, there is no significant difference of age between AMS group and Non-AMS individuals in microRNA array screening set $(23 \pm 2.5$ vs. $26 \pm 6.5, P=0.071)$ and qRTPCR assay set ( $22 \pm 3.5$ vs. $22 \pm 4.0, P=0.081)$, as well as body mass index between AMS and Non-AMS groups (microRNA array screening set: $21 \pm 2.5$ vs. $22 \pm 3.0, P=0.475$; qRTPCR assay set: $22 \pm 2.0$ vs. $21 \pm 4.0, P=0.370$; Table 2 ). After exposure to high altitude, oxygen saturation of all individuals significantly decreased, while heart rate significantly increased.

TABLE 2 | Characteristics of subjects.

\begin{tabular}{|c|c|c|c|}
\hline Characteristics & AMS & Non-AMS & $P$-values \\
\hline \multicolumn{4}{|c|}{ microRNAS ARRAY SCREENING SET $(n=22)$} \\
\hline \multicolumn{4}{|l|}{ Age (Year) } \\
\hline Median $\pm I Q R^{*}$ & $23 \pm 2.5$ & $26 \pm 6.5$ & 0.071 \\
\hline Range & $24-32$ & $24-35$ & \\
\hline \multicolumn{4}{|l|}{ Sex } \\
\hline Male & 13 & 9 & \\
\hline Female & 0 & 0 & \\
\hline \multicolumn{4}{|l|}{ BMI $^{*}\left(\mathbf{k g} / \mathbf{m}^{2}\right)$} \\
\hline Median $\pm I Q R^{\star}$ & $21 \pm 2.5$ & $22 \pm 3.0$ & 0.475 \\
\hline Range & $20-25$ & 19-26 & \\
\hline \multicolumn{4}{|c|}{ qRT-PCR ASSAY SET $(n=87)$} \\
\hline \multicolumn{4}{|l|}{ Age (Year) } \\
\hline Median $\pm I Q R^{*}$ & $22 \pm 3.5$ & $22 \pm 4.0$ & 0.081 \\
\hline Range & $17-27$ & $17-26$ & \\
\hline \multicolumn{4}{|l|}{ Sex } \\
\hline Male & 41 & 46 & \\
\hline Female & 0 & 0 & \\
\hline \multicolumn{4}{|l|}{$\mathrm{BMI}^{*}\left(\mathbf{k g} / \mathbf{m}^{2}\right)$} \\
\hline Median \pm IQR $^{*}$ & $22 \pm 2.0$ & $21 \pm 4.0$ & 0.370 \\
\hline Range & $18-25$ & $18-26$ & \\
\hline
\end{tabular}

${ }^{*} / Q R$, interquartile range; BMI, body mass index. 
However, only on the third day after exposure to high altitude, heart rate of AMS group was higher than Non-AMS individuals (Supplementary Table 2).

\section{MicroRNA Array and Validation of microRNAs}

MicroRNA array screening revealed that 31 microRNAs were differentially expressed between AMS and Non-AMS groups, 15 up-regulated and 16 down-regulated (Figure 2A). Among them, miR-369-3p, miR-449b-3p, miR-136-3p, and miR-4791 (MIMAT0019963) (all Fold changes > 5) were the most significantly up-regulated microRNAs in the AMS group compared with Non-AMS individuals. All the four microRNAs were considered for further validation with qRT-PCR assay.

Consistently, miR-369-3p, miR-449b-3p, miR-136-3p, and miR-4791 (all $P<0.001$ ) were significantly up-regulated in AMS patients compared to Non-AMS individuals, using cel-miR-39 as normalization control, within qRT-PCR test (Figure 2B).

\section{MicroRNA Signature for the Identification of Patients with Acute Mountain Sickness}

The area under the curve (AUC) of miR-369-3p, miR-449b$3 \mathrm{p}$, miR-136-3p, and miR-4791 was 0.859, 0.847, 0.724, and 0.766, respectively (Figure 3, Table 3). Among them, miR-369-3p present the highest accuracy for discrimination AMS from NonAMS individuals. However, the AUC was $<0.850$ for the remind single-microRNA to predict AMS.

Combination of markers could improve accuracy in disease diagnosis (Pepe and Thompson, 2000). So we performed receiver operating characteristic curves for combination of miR-369-3p, miR-449b-3p, miR-136-3p, and miR-4791 with Logistic Regression analysis. A binomial logistic regression was performed to ascertain the effects of miR-369-3p, miR-449b-3p, miR-136-3p, and miR-4791 on the likelihood that participants have AMS. Of the four predictor variables only three were statistically significant: miR-369-3p, miR-449b-3p, and miR-1363p (Supplementary Table 3). Therefore, miR-4791 was excluded from the logistic regression model.

The logistic regression model for miR-369-3p, miR-449b-3p, and miR-136-3p was statistically significant, $\chi_{(3)}^{2}=95.616, P$ $<0.001$. The model explained $89.0 \%$ (Nagelkerke $R^{2}$ ) of the variance in AMS and correctly classified $94.3 \%$ of cases. All predictor variables were statistically significant (Supplementary Table 4). Notably, the combination of miR-369-3p, miR$449 \mathrm{~b}-3 \mathrm{p}$, and miR-136-3p resulted in a robustly increased AUC (0.986, 95\%CI 0.970-1.000; LR+: 14.21; LR-: 0.08), leading to a signature for the prediction of AMS (Figure 4). Accordingly, despite the single-microRNA cut-off values between AMS and Non-AMS (>0.038 for miR-369-3p, >29.602 for miR-449b-3p, and $>0.045$ for miR-136-3p; Table 3), a global cut-off value of $>0.110$ for the signature of miR-369-3p, miR-449b-3p, and miR-136-3p combination increased the diagnostic power to a $92.68 \%$ sensitivity and a $93.48 \%$ specificity.
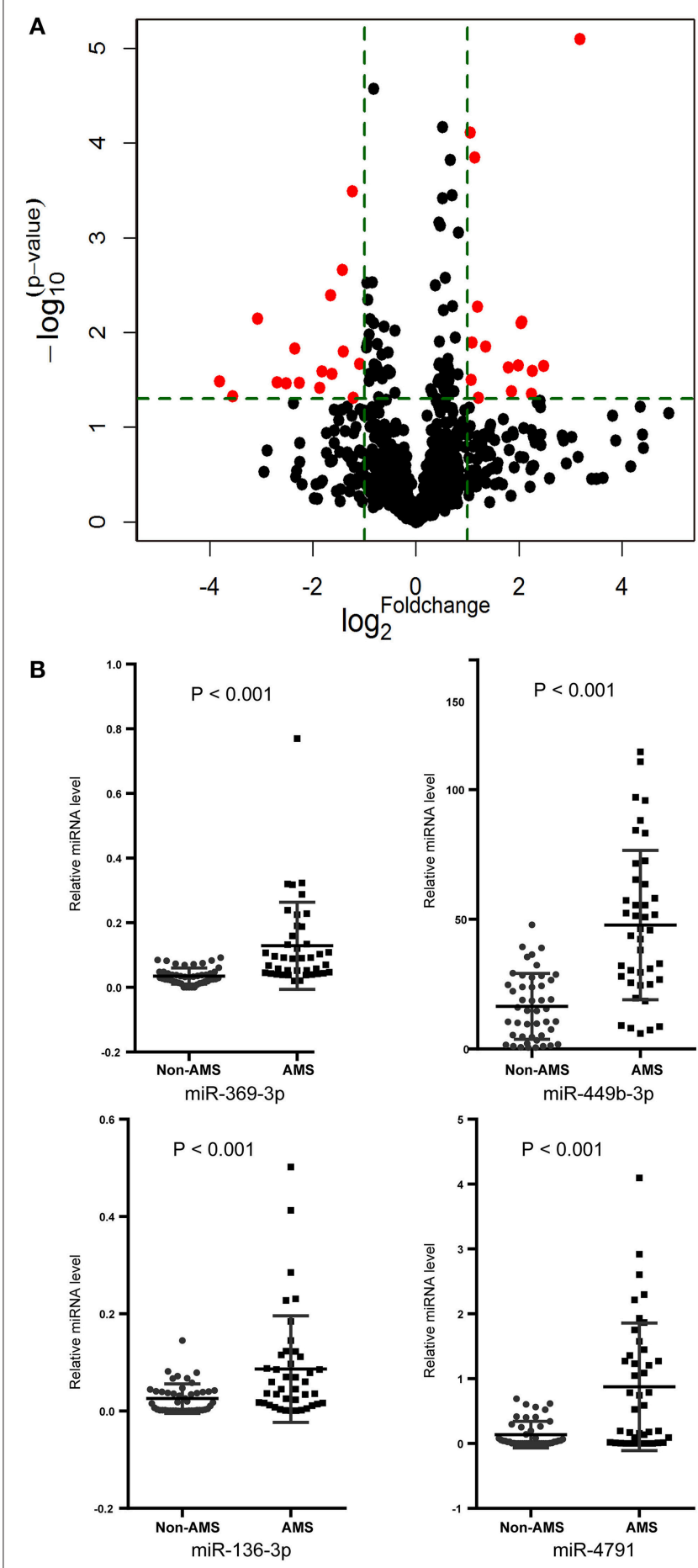

FIGURE 2 | Circulating microRNAs expression profile was different between acute mountain sickness (AMS) and non-acute mountain sickness (Non-AMS) groups. (A) Comparisons of all microRNAs in microarray analysis of RNA isolated from plasma of AMS and Non-AMS groups. The volcano plot displays the relationship between fold-change and significance using a scatter plot view. The red points in the plot represent the differentially expressed microRNAs with statistical significance. (B) Results of the quantitative reverse-transcription polymerase chain reaction of the miR-369-3p, miR-449b-3p, miR-136-3p, and miR-4791 expression (AMS, $n$ $=41$; Non-AMS, $n=46$ ). 


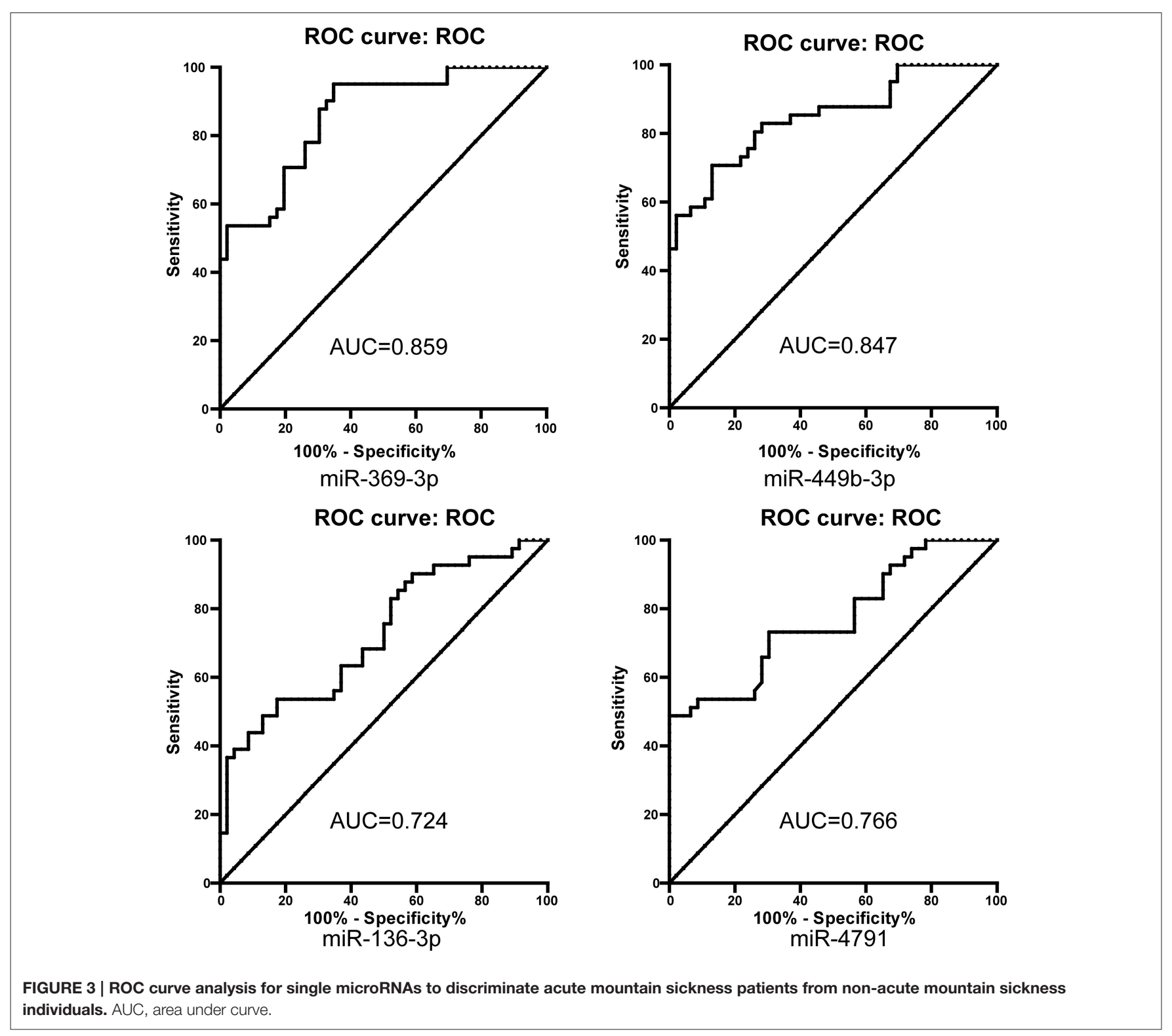

TABLE 3 | Receiver operating characteristic curves of single-plasma microRNAs.

\begin{tabular}{lcccccccccccc}
\hline & AUC & $\mathbf{9 5} \% \mathbf{C l}$ & $\boldsymbol{P}$-values & OR per SD & $\mathbf{9 5 \%} \mathbf{C l}$ & Cut off & Sensitivity (\%) & $\mathbf{9 5 \%} \mathbf{C l}$ & Specificity (\%) & $\mathbf{9 5 \%} \mathbf{C l}$ & $\mathbf{L R +}$ & $\mathbf{L R}-$ \\
\hline AMS vs. Non-AMS & & & & & & & & & & & & \\
miR-369-3p & 0.859 & $0.783-0.935$ & $<0.001$ & 62.586 & $8.032-487.699$ & $>0.038$ & 0.95 & $0.83-0.99$ & 0.65 & $0.50-0.79$ & 2.73 & 0.07 \\
miR-449b-3p & 0.847 & $0.765-0.929$ & $<0.001$ & 8.659 & $3.311-22.648$ & $>29.602$ & 0.71 & $0.54-0.84$ & 0.87 & $0.74-0.95$ & 5.42 & 0.33 \\
miR-136-3p & 0.724 & $0.617-0.831$ & $<0.001$ & 4.874 & $1.740-13.433$ & $>0.045$ & 0.54 & $0.37-0.69$ & 0.83 & $0.69-0.92$ & 3.08 & 0.56 \\
miR-4791 & 0.766 & $0.666-0.867$ & $<0.001$ & 7.130 & $2.409-21.104$ & $>0.091$ & 0.73 & $0.57-0.86$ & 0.70 & $0.54-0.82$ & 2.40 & 0.39 \\
\hline
\end{tabular}

AUC, area under curve; $\mathrm{Cl}$, confidence interval; $L R+$, positive likelihood ratio; $L R$-, negative likelihood ratio; OR per SD, odds ratio per standard deviation; AMS, acute mountain sickness.

\section{Biological Relevance of microRNA}

\section{Signature}

GO enrichment analysis for target genes of microRNA signature revealed that cellular nitrogen compound metabolic process, gene expression, neurotrophin TRK receptor signaling pathway, biosynthetic process, and viral process were the top five GO term over-represented in target genes of the microRNA signature (Figure 5, Supplementary Table 5). 


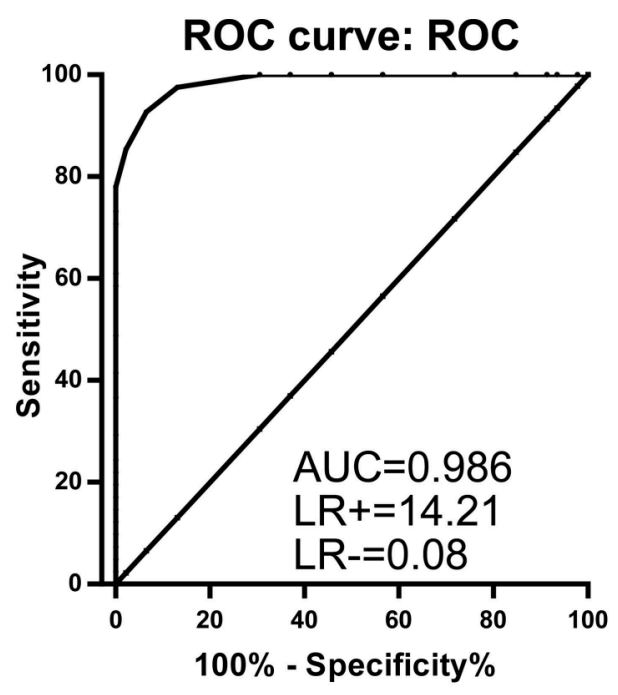

FIGURE 4 | ROC curve analysis for the combination of three microRNAs (miR-369-3p, miR-449b-3p, and miR-136-3p) resulting in enhanced specificity and sensitivity to distinguish acute mountain sickness patients from non-acute mountain sickness individuals (97.6, 93.4\%, respectively). AUC, area under curve; LR, likelihood ratio.

\section{DISCUSSION}

The number of individuals who ascended to high altitude for a variety of reasons is increasing. AMS which can progress to high altitude cerebral edema is a common problem to them (Honigman et al., 1993; Hackett and Roach, 2001; Karinen et al., 2008). Speed of ascent (Hackett et al., 1976), previous history of AMS (Schneider et al., 2002), obesity (Ri-Li et al., 2003), migraine (Mairer et al., 2009), and so forth (Taylor, 2011) have been identified as risk factors for AMS. However, the incidence of AMS is different in individuals, families and populations because of their different capacity to cope with high-altitude environments (MacInnis et al., 2010). This study for the first time reports a novel signature of three circulating microRNAs allowing to distinguishing AMS from Non-AMS individuals before exposure to high altitude.

Nowadays, there are many ways to prevent AMS through non-pharmacologic and pharmacologic measures (Imray et al., 2010). Providing time for the body to acclimatize to high altitude is the single best way to prevent AMS (Beidleman et al., 2004; Luks et al., 2014), but it is not impracticable to individuals with urgent task. Pharmacologic prophylaxis is an appropriate way for the majority of individuals; however, the adverse effect of pharmaceutical products could impair the health of people (Dumont et al., 2000). Thus, identifying individuals susceptible to AMS would assist in the development of suitable preventive strategies for a given individual.

The study here provided a more effective method to detect individuals at risk of AMS with circulating microRNAs than those used currently. The receiver operating characteristic curves revealed that the power of diagnostic test for AMS with a $92.68 \%$ sensitivity and a $93.48 \%$ specificity. Although already one microRNA could discriminate to AUC of 0.859 between the different groups, this approach failed in appropriate LR. Only the combination of three microRNAs managed to deliver an increased discrimination (AUC 0.986) with a high positive LR in accordance with a low negative LR.

The biological relevance of circulating microRNAs is now regarded as a global, hormone-like functional molecule that might allow regulation of gene expression across tissues at a distance (Turchinovich et al., 2013). Here, we found that the expression value of microRNA signature in AMS susceptible individuals was significantly higher than AMS resistant individuals. Its target genes more enriched in cellular nitrogen compound metabolic process and neurotrophin TRK receptor signaling pathway. Nitric oxide (NO), generated through cellular nitrogen compound metabolic process, is a major signaling and effector molecule mediating the body's response to hypoxia (Beall et al., 2012). The generation of high levels of NO and circulating nitrogen oxide species promote the adaptation of natives of Tibet and acclimatization of lowlanders who exposure to high altitude via enabling greater blood flow and oxygen delivery (Erzurum et al., 2007; Janocha et al., 2011). Neurotrophin TRK receptor is high-affinity receptor of brain-derived neurotrophic factor whose concentration will be increased in human plasma after exposure to high altitude (elevation: $3350 \mathrm{~m}$ ) for $72 \mathrm{~h}$ (Helan et al., 2014). Prakash and his colleagues confirmed that brain-derived neurotrophic factor could induce NO generation in human pulmonary artery endothelial cells via TRK receptor (Meuchel et al., 2011).

Previously studies affirm that high levels of NO are associated with function benefits and the avoidance of illness (Janocha et al., 2011). The higher expression value of microRNA signature in AMS susceptible individuals will suppress the genes involved in cellular nitrogen compound metabolic process and neurotrophin TRK receptor signaling pathway, post-transcriptionally and cause a low levels of $\mathrm{NO}$ and circulating nitrogen oxide species. Thus, the blunt production of $\mathrm{NO}$ and circulating nitrogen oxide species via microRNAs may be the biological underpinning of AMS susceptible individuals who present a higher expression level of the microRNA signature consisted of miR-369-3p, miR449b-3p, and miR-136-3p.

We demonstrated for the first time a unique signature of circulating microRNAs to differentiate AMS from Non-AMS individuals. However, these results need to be confirmed in a large patient cohort to exclude potential bias. And only the young health men were included in this study because they are the main part of population who travel to high altitudes for recreation, work, and pilgrimage. Therefore, further investigations in more individuals, also including females, other races and ages, should be obtained to affirm the performance of the signature proposed here.

\section{CONCLUSION}

Here, we, for the first time, describe a signature of circulating microRNAs for sensitive and specific identification of the susceptibility of AMS before exposure to high altitude. This 


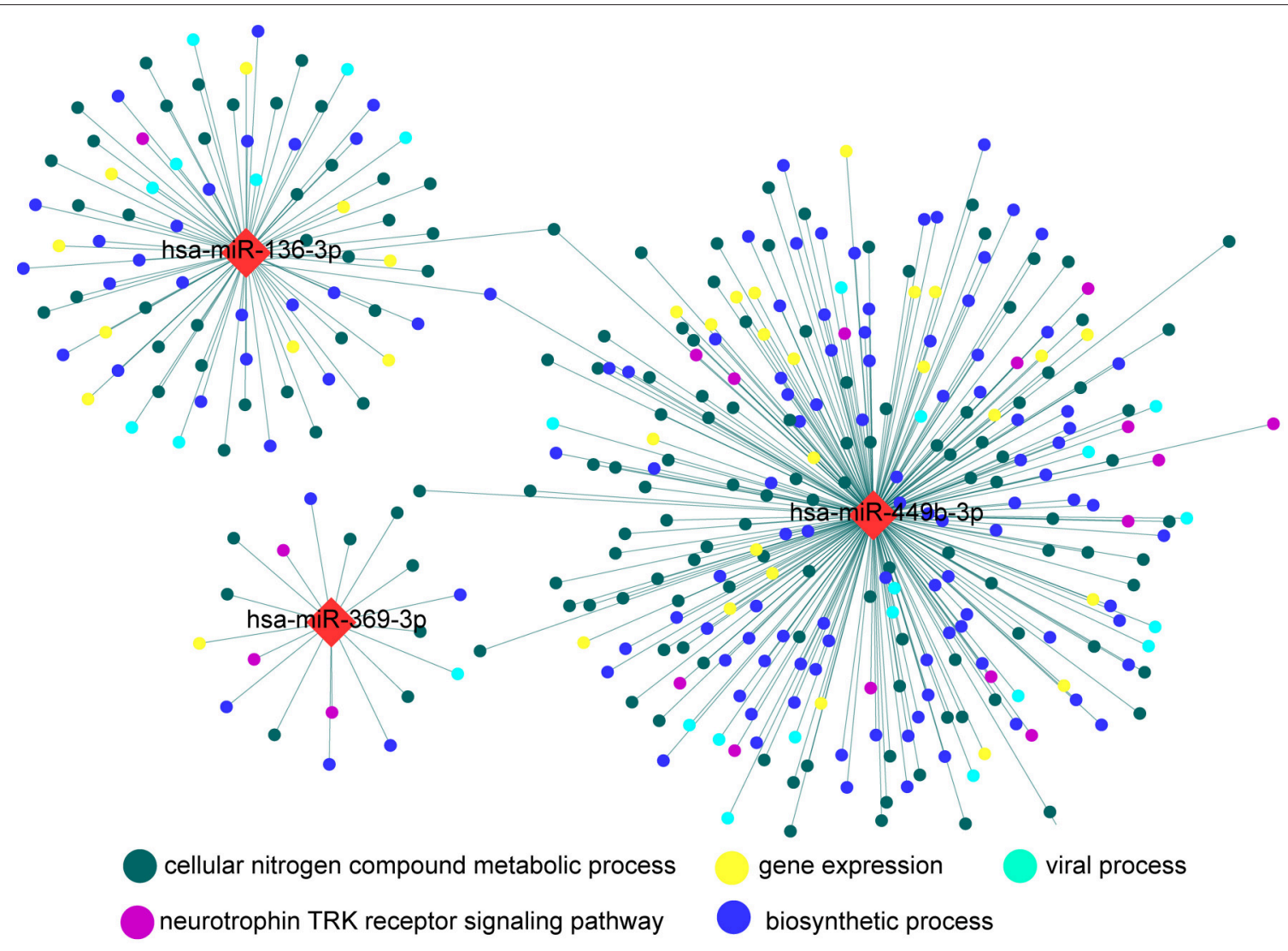

FIGURE 5 | Direct interaction network between microRNAs and target genes. The diamond nodes represent microRNAs, while circular nodes represent target genes. Color of circular nodes represents the most enriched association with a top five GO biological process. GO, Gene ontology.

signature may hold great promise to become an important tool for the prediction of AMS.

\section{AVAILABILITY OF DATA AND MATERIALS}

All data have been submitted to GEO under the accession GSE90500.

\section{AUTHOR CONTRIBUTIONS}

YG conceived and designed the study. $\mathrm{HH}$ and JC oversaw laboratory analyses and $\mathrm{BL}$ provided the overall supervision of the study. GW, EZ, and GX did the laboratory experiments or contributed the statistical analysis, or both GW and BS contributed to sample and physical data collections. BL drafted the report. All authors contributed

\section{REFERENCES}

Ai, J., Zhang, R., Li, Y., Pu, J., Lu, Y., Jiao, J., et al. (2010). Circulating microRNA1 as a potential novel biomarker for acute myocardial infarction. Biochem. Biophys. Res. Commun. 391, 73-77. doi: 10.1016/j.bbrc.2009.11.005 to the interpretation of results, critical revision of the manuscript, and approved the final manuscript. YG is the guarantor.

\section{ACKNOWLEDGMENTS}

This work was supported by the Key Projects in the Military Science and Technology Pillar Program during the 13 fiveyear Plan Period (AWS14C007), by National Natural Science Foundation of China (J1310001).

\section{SUPPLEMENTARY MATERIAL}

The Supplementary Material for this article can be found online at: http://journal.frontiersin.org/article/10.3389/fphys. 2017.00055/full\#supplementary-material

Akhtar, M. M., Micolucci, L., Islam, M. S., Olivieri, F., and Procopio, A. D. (2016) Bioinformatic tools for microRNA dissection. Nucleic Acids Res. 44, 24-44. doi: 10.1093/nar/gkv1221

Bärtsch, P., and Swenson, E. R. (2013). Clinical practice: acute high-altitude illnesses. N.Engl. J. Med. 368, 2294-2302. doi: 10.1056/NEJMcp1214870 
Basnyat, B., and Murdoch, D. R. (2003). High-altitude illness. Lancet 361, 1967-1974. doi: 10.1016/S0140-6736(03)13591-X

Beall, C. M., Laskowski, D., and Erzurum, S. C. (2012). Nitric oxide in adaptation to altitude. Free Radic. Biol. Med. 52, 1123-1134. doi: 10.1016/j.freeradbiomed.2011.12.028

Beidleman, B. A., Muza, S. R., Fulco, C. S., Cymerman, A., Ditzler, D., Stulz, D., et al. (2004). Intermittent altitude exposures reduce acute mountain sickness at 4300 m. Clin. Sci. 106, 321-328. doi: 10.1042/CS20030161

Cline, M. S., Smoot, M., Cerami, E., Kuchinsky, A., Landys, N., Workman, C., et al. (2007). Integration of biological networks and gene expression data using Cytoscape. Nat. Protoc. 2, 2366-2382. doi: 10.1038/nprot.2007.324

Ding, H., Liu, Q., Hua, M., Ding, M., Du, H., Zhang, W., et al. (2011). Polymorphisms of hypoxia-related genes in subjects susceptible to acute mountain sickness. Respiration 81, 236-241. doi: 10.1159/000322850

Dumont, L., Mardirosoff, C., and Tramèr, M. R. (2000). Efficacy and harm of pharmacological prevention of acute mountain sickness: quantitative systematic review. BMJ 321, 267-272. doi: 10.1136/bmj.321.7256.267

Dumortier, O., Hinault, C., and Van Obberghen, E. (2013). MicroRNAs and metabolism crosstalk in energy homeostasis. Cell Metab. 18, 312-324. doi: 10.1016/j.cmet.2013.06.004

Erzurum, S. C., Ghosh, S., Janocha, A. J., Xu, W., Bauer, S., Bryan, N. S., et al. (2007). Higher blood flow and circulating NO products offset high-altitude hypoxia among Tibetans. Proc. Natl. Acad. Sci. U.S.A. 104, 17593-17598. doi: 10.1073/pnas.0707462104

Gasparini, G., Vicini, C., De Benedetto, M., Salamanca, F., Sorrenti, G., Romandini, M., et al. (2015). Diagnostic accuracy of obstructive airway adult test for diagnosis of obstructive sleep Apnea. Biomed. Res. Int. 2015:915185. doi: 10.1155/2015/915185

Hackett, P. H., Rennie, D., and Levine, H. D. (1976). The incidence, importance, and prophylaxis of acute mountain sickness. Lancet 2, 1149-1155. doi: 10.1016/S0140-6736(76)91677-9

Hackett, P. H., and Roach, R. C. (2001). High-altitude illness. N.Engl. J. Med. 345, 107-114. doi: 10.1056/NEJM200107123450206

Hackett, P. H., and Roach, R. C. (2004). High altitude cerebral edema. High Alt. Med. Biol. 5, 136-146. doi: 10.1089/1527029041352054

Helan, M., Aravamudan, B., Hartman, W. R., Thompson, M. A., Johnson, B. D., Pabelick, C. M., et al. (2014). BDNF secretion by human pulmonary artery endothelial cells in response to hypoxia. J. Mol. Cell. Cardiol. 68, 89-97. doi: 10.1016/j.yjmcc.2014.01.006

Honigman, B., Theis, M. K., Koziol-Mclain, J., Roach, R., Yip, R., Houston, C., et al. (1993). Acute mountain sickness in a general tourist population at moderate altitudes. Ann. Intern. Med. 118, 587-592. doi: 10.7326/0003-4819-118-8-199304150-00003

Imray, C., Wright, A., Subudhi, A., and Roach, R. (2010). Acute mountain sickness: pathophysiology, prevention, and treatment. Prog. Cardiovasc. Dis. 52, 467-484. doi: 10.1016/j.pcad.2010.02.003

Janocha, A. J., Koch, C. D., Tiso, M., Ponchia, A., Doctor, A., Gibbons, L., et al. (2011). Nitric oxide during altitude acclimatization. N.Engl. J. Med. 365, 1942-1944. doi: 10.1056/NEJMc1107887

Karinen, H. M., Uusitalo, A., Vähä-Ypyä H., Kähönen, M., Peltonen, J. E., Stein, P. K., et al. (2012). Heart rate variability changes at $2400 \mathrm{~m}$ altitude predicts acute mountain sickness on further ascent at $3000-4300 \mathrm{~m}$ altitudes. Front. Physiol. 3:336. doi: $10.3389 /$ fphys.2012.00336

Karinen, H., Peltonen, J., and Tikkanen, H. (2008). Prevalence of acute mountain sickness among Finnish trekkers on Mount Kilimanjaro, Tanzania: an observational study. High Alt. Med. Biol. 9, 301-306. doi: $10.1089 /$ ham. 2008.1008

Koehle, M. S., Guenette, J. A., and Warburton, D. E. (2010). Oximetry, heart rate variability, and the diagnosis of mild-to-moderate acute mountain sickness. Eur. J. Emerg. Med. 17, 119-122. doi: 10.1097/MEJ.0b013e32832fa099

Kovtun, L. T., and Voevoda, M. I. (2013). Susceptibility to hypoxia and breathing control changes after short-term cold exposures. Int. J. Circumpolar Health 72. doi: $10.3402 /$ ijch.v72i0.21574

Lenkala, D., Lacroix, B., Gamazon, E. R., Geeleher, P., Im, H. K., and Huang, R. S. (2014). The impact of microRNA expression on cellular proliferation. Hum. Genet. 133, 931-938. doi: 10.1007/s00439-014-1434-4

Liu, B., Huang, H., Wang, S.-X., Wu, G., Xu, G., Sun, B.-D., et al. (2016). Physiological adjustments and circulating MicroRNA reprogramming are involved in early acclimatization to high altitude in Chinese Han Males. Front. Physiol. 7:601. doi: 10.3389/fphys.2016.00601

López-Ratón, M., Rodríguez-Âlvarez, M. X., Cadarso-Suárez, C., and GudeSampedro, F. (2014). OptimalCutpoints: an R package for selecting optimal cutpoints in diagnostic tests. J. Stat. Softw. 61, 1-36. doi: 10.18637/jss.v061.i08

Luks, A. M., McIntosh, S. E., Grissom, C. K., Auerbach, P. S., Rodway, G. W., Schoene, R. B., et al. (2014). Wilderness medical society practice guidelines for the prevention and treatment of acute altitude illness: 2014 update. Wilderness Environ. Med. 25, S4-S14. doi: 10.1016/j.wem.2014.06.017

MacInnis, M. J., Carter, E. A., Freeman, M. G., Pandit, B. P., Siwakoti, A., Subedi, A., et al. (2013). A prospective epidemiological study of acute mountain sickness in Nepalese pilgrims ascending to high altitude $(4380 \mathrm{~m})$. PLoS ONE 8:e75644. doi: 10.1371/journal.pone.0075644

MacInnis, M. J., Koehle, M. S., and Rupert, J. L. (2010). Evidence for a genetic basis for altitude illness: 2010 update. High Alt. Med. Biol. 11, 349-368. doi: 10.1089/ham.2010.1030

Maggiorini, M., Müller, A., Hofstetter, D., Bärtsch, P., and Oelz, O. (1998). Assessment of acute mountain sickness by different score protocols in the Swiss Alps. Aviat. Space Environ. Med. 69, 1186-1192.

Mairer, K., Wille, M., Bucher, T., and Burtscher, M. (2009). Prevalence of acute mountain sickness in the Eastern Alps. High Alt. Med. Biol. 10, 239-245. doi: 10.1089/ham.2008.1091

Meuchel, L. W., Thompson, M. A., Cassivi, S. D., Pabelick, C. M., and Prakash, Y. S. (2011). Neurotrophins induce nitric oxide generation in human pulmonary artery endothelial cells. Cardiovasc. Res. 91, 668-676. doi: 10.1093/cvr/cvr107

Mitchell, P. S., Parkin, R. K., Kroh, E. M., Fritz, B. R., Wyman, S. K., PogosovaAgadjanyan, E. L., et al. (2008). Circulating microRNAs as stable blood-based markers for cancer detection. Proc. Natl. Acad. Sci. U.S.A. 105, 10513-10518. doi: $10.1073 /$ pnas. 0804549105

Moussay, E., Wang, K., Cho, J. H., van Moer, K., Pierson, S., Paggetti, J., et al. (2011). MicroRNA as biomarkers and regulators in B-cell chronic lymphocytic leukemia. Proc. Natl. Acad. Sci. U.S.A. 108, 6573-6578. doi: $10.1073 /$ pnas. 1019557108

Paraskevopoulou, M. D., Georgakilas, G., Kostoulas, N., Vlachos, I. S., Vergoulis, T., Reczko, M., et al. (2013). DIANA-microT web server v5.0: service integration into miRNA functional analysis workflows. Nucleic Acids Res. 41, W169-W173. doi: 10.1093/nar/gkt393

Pennardt, A. (2013). High-altitude pulmonary edema: diagnosis, prevention, and treatment. Curr. Sports Med. Rep. 12, 115-119. doi: 10.1249/ ISR.0b013e318287713b

Pepe, M. S., and Thompson, M. L. (2000). Combining diagnostic test results to increase accuracy. Biostatistics 1, 123-140. doi: 10.1093/biostatistics/1.2.123

Ri-Li, G., Chase, P. J., Witkowski, S., Wyrick, B. L., Stone, J. A., Levine, B. D., et al. (2003). Obesity: associations with acute mountain sickness. Ann. Intern. Med. 139, 253-257. doi: 10.7326/0003-4819-139-4-200308190-00007

Roach, R. C., Bartsch, P., Hackett, P. H., and Oelz, O.,(1993). "The lake louise acute mountain sickness scoring system," in Proceedings of the 8th International Hypoxia Symposium, Lake Louise, Alberta, Canada, Hypoxia and Molecular Medicine, eds J. R Sutton, G. Coates, and C. S. Houston (Burlington, VT: Queen City Printers), 272-274

Roth, P., Wischhusen, J., Happold, C., Chandran, P. A., Hofer, S., Eisele, G., et al. (2011). A specific miRNA signature in the peripheral blood of glioblastoma patients. J. Neurochem. 118, 449-457. doi: 10.1111/j.1471-4159.2011.07307.x

Schneider, M., Bernasch, D., Weymann, J., Holle, R., and Bartsch, P. (2002). Acute mountain sickness: influence of susceptibility, preexposure, and ascent rate. Med. Sci. Sports Exerc. 34, 1886-1891. doi: 10.1097/00005768-20021200000005

Shivdasani, R. A. (2006). MicroRNAs: regulators of gene expression and cell differentiation. Blood 108, 3646-3653. doi: 10.1182/blood-2006-01-030015

Song, H., Ke, T., Luo, W. J., and Chen, J. Y. (2013). Non-high altitude methods for rapid screening of susceptibility to acute mountain sickness. BMC Public Health 13:902. doi: 10.1186/1471-2458-13-902

Su, Z., Yang, Z., Xu, Y., Chen, Y., and Yu, Q. (2015). MicroRNAs in apoptosis, autophagy and necroptosis. Oncotarget 6, 8474-8490. doi: 10.18632/oncotarget.3523

Taylor, A. T. (2011). High-altitude illnesses: physiology, risk factors, prevention, and treatment. Rambam Maimonides Med. J. 2:e0022. doi: $10.5041 /$ RMMJ.10022 
Turchinovich, A., Samatov, T. R., Tonevitsky, A. G., and Burwinkel, B. (2013). Circulating miRNAs: cell-cell communication function? Front. Genet. 4:119. doi: 10.3389/fgene.2013.00119

Vlachos, I. S., Paraskevopoulou, M. D., Karagkouni, D., Georgakilas, G., Vergoulis, T., Kanellos, I., et al. (2015a). DIANA-TarBase v7.0: indexing more than half a million experimentally supported miRNA:mRNA interactions. Nucleic Acids Res. 43, D153-D159. doi: 10.1093/nar/gk u1215

Vlachos, I. S., Zagganas, K., Paraskevopoulou, M. D., Georgakilas, G., Karagkouni, D., Vergoulis, T., et al. (2015b). DIANA-miRPath v3.0: deciphering microRNA function with experimental support. Nucleic Acids Res. 43, W460-W466. doi: $10.1093 /$ nar/gkv403

Waeber, B., Kayser, B., Dumont, L., Lysakowski, C., Tramèr, M. R., and Elia, N. (2015). Impact of Study Design on reported incidences of acute mountain sickness: a systematic review. High Alt. Med. Biol. 16, 204-215. doi: 10.1089/ham.2015.0022

Weber, J. A., Baxter, D. H., Zhang, S., Huang, D. Y., Huang, K. H., Lee, M. J., et al. (2010). The microRNA spectrum in 12 body fluids. Clin. Chem. 56, 1733-1741. doi: $10.1373 /$ clinchem.2010.147405
Wright, A., Brearey, S., and Imray, C. (2008). High hopes at high altitudes: pharmacotherapy for acute mountain sickness and high-altitude cerebral and pulmonary oedema. Expert Opin. Pharmacother. 9, 119-127. doi: 10.1517/14656566.9.1.119

Zhou, Q. Q., Gao, Y. Q., and Huang, Q. Y. (2004). Predictive effect of lung functional determination of the population susceptible to acute mountain sickness. Med. J. Nat. Def. For. 25, 13-15.

Conflict of Interest Statement: The authors declare that the research was conducted in the absence of any commercial or financial relationships that could be construed as a potential conflict of interest.

Copyright $\odot 2017$ Liu, Huang, Wu, Xu, Sun, Zhang, Chen and Gao. This is an open-access article distributed under the terms of the Creative Commons Attribution License (CC BY). The use, distribution or reproduction in other forums is permitted, provided the original author(s) or licensor are credited and that the original publication in this journal is cited, in accordance with accepted academic practice. No use, distribution or reproduction is permitted which does not comply with these terms. 